\title{
Reducing childhood respiratory morbidity and mortality in low and middle income countries: a current challenge
}

\author{
Cristina Ardura-Garcia (10 ${ }^{1}$ and Claudia E. Kuehni (10), \\ Affiliations: ${ }^{1}$ Institute of Social and Preventive Medicine, University of Bern, Bern, Switzerland. ${ }^{2}$ Paediatric \\ Respiratory Medicine, Children's University Hospital of Bern, University of Bern, Bern, Switzerland. \\ Correspondence: Claudia E. Kuehni; Institute of Social and Preventive Medicine, University of Bern, \\ Mittelstrasse 43, 3012 Bern, Switzerland. E-mail: claudia.kuehnidispm.unibe.ch
}

@ERSpublications

Pneumonia overdiagnosis and asthma underdiagnosis in children should be addressed together in low resource settings http://bit.ly/2XPvega

Cite this article as: Ardura-Garcia C, Kuehni CE. Reducing childhood respiratory morbidity and mortality in low and middle income countries: a current challenge. Eur Respir J 2019; 54: 1900987 [https://doi.org/ 10.1183/13993003.00987-2019].

\section{Introduction}

Pneumonia is a major cause of death of children in low and middle income countries (LMICs). In this issue of the European Respiratory Journal, NGUYEN et al. [1] examine pneumonia care in a large secondary care hospital in Vietnam and compare it to World Health Organization (WHO) case management guidelines. This carefully conducted prospective study highlights the weaknesses of the WHO approach, which leads to overdiagnosis of pneumonia with unnecessary hospitalisations and antibiotic use, and underdiagnosis of obstructive airway diseases.

\section{Pneumonia: major killer in children worldwide}

Pneumonia remains a major cause of death worldwide in children beyond the neonatal period and under 5 years of age [2], and is a major cause of hospitalisation and morbidity in children and adults in LMICs. Interventions, including preventive measures such as the Haemophilus influenza type b and pneumococcus conjugate vaccines, and management strategies, such as the WHO Integrated Management of Childhood Illness (IMCI) guidelines, have brought about a steady decline in pneumonia incidence and mortality $[3,4]$. Despite these efforts, 800000 children aged under 5 years old, most of them in LMICs, died in 2017 from a lower respiratory infection [2]. This is an alarming number because adequate management would prevent most of these deaths.

Management of community-acquired pneumonia is based on clinical diagnosis and antibiotic treatment, with further radiological and laboratory tests and supportive therapy for those admitted to hospital $[5,6]$. However, not all diagnostic procedures are readily available in LMICs, where diagnosis focuses on medical history and examination. This may lead to overdiagnosis of pneumonia and unnecessary use of antibiotics and hospital resources [7]. In an attempt to reduce pneumonia mortality and unnecessary antibiotic use in children, WHO developed a case management approach for low resource settings such as the one included in the IMCI guidelines $[3,8]$. It consists of the identification of children with cough and/or difficulty breathing and their categorisation into cough/cold without pneumonia, simple pneumonia, or severe pneumonia. Pneumonia is classified as simple based on the presence of fast breathing alone, and as severe 


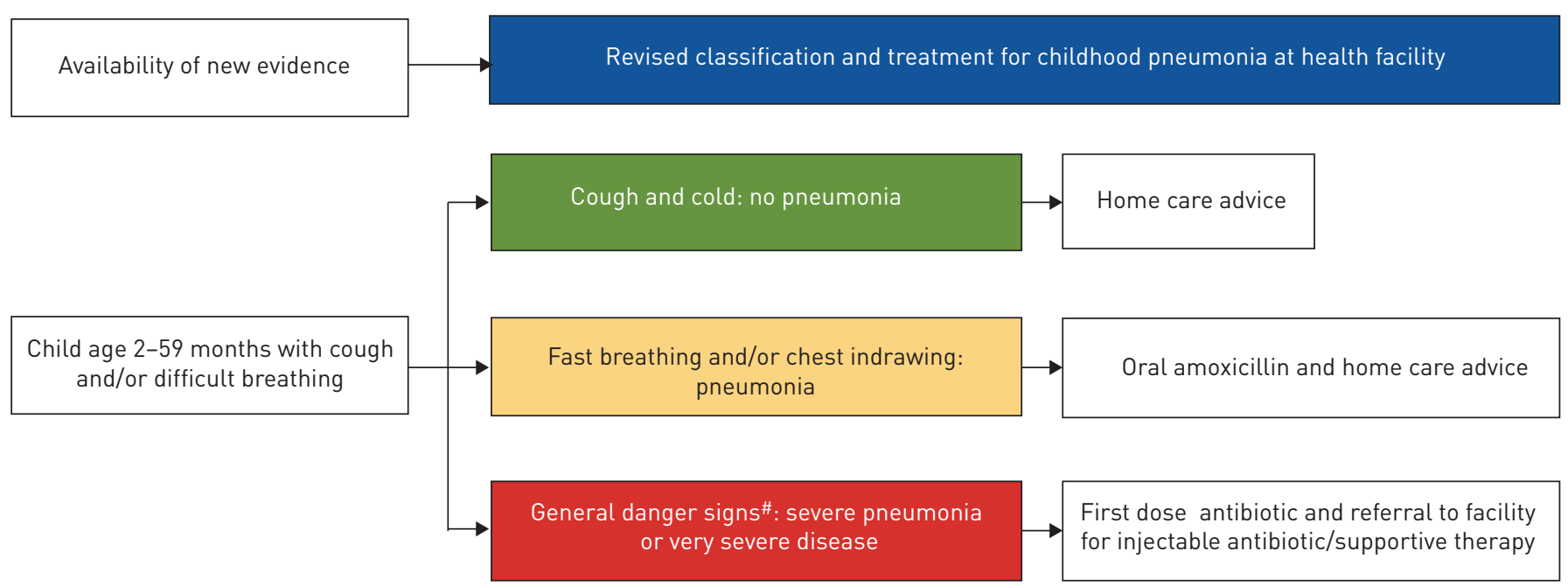

FIGURE 1 World Health Organization 2014 revised classification and treatment for children 2-59 months old presenting with cough and/or difficulty breathing. " : not able to drink, persistent vomiting, convulsions, lethargic or unconscious, stridor in a calm child or severe malnutrition. Reproduced with permission from the Revised WHO classification and treatment of pneumonia in children at health facilities: evidence summaries, World Health Organization, 2014 [12].

or very severe if children also have chest indrawing, stridor, and/or danger signs such as low peripheral oxygen saturation, inability to drink, or lethargy. Those diagnosed with simple pneumonia receive oral antibiotics and home care, while those with severe pneumonia are admitted to hospital to receive intravenous antibiotics. This strategy has been applied in multiple low resource settings and several studies highlight its strengths and limitations [9-11]. Its classifications were revised in 2014 by the addition of chest indrawing as indicative of simple pneumonia (figure 1) [12].

A meta-analysis of Asian and African studies suggests that a case management approach may reduce all-cause mortality by $24 \%$ and pneumonia mortality by $36 \%$ in $0-4$ year olds [9]. Because $80 \%$ of pneumonia deaths occur out of hospital [13], the use of specific community-focused case management programmes may have the greatest impact on mortality. A 2010 review showed a $70 \%$ reduction in pneumonia mortality in children aged under 5 years when applying community case management strategies [10].

The WHO case management strategies were developed mainly to help community healthcare workers identify children with pneumonia using only clinical signs. The strategies are highly sensitive but less specific, and diagnose as pneumonia any lower respiratory disease presenting with cough, difficulty breathing and tachypnoea. Viral diseases like bronchiolitis or viral wheeze, and non-communicable diseases like allergic asthma will be diagnosed and treated as pneumonia, resulting in overprescription of antibiotics [14] and underdiagnosis and inadequate management of obstructive airway disease. The 2014 pneumonia case management revision aimed at improving pneumonia outcomes and decreasing the number of children hospitalised by classifying children with chest indrawing as having simple pneumonia [12] instead of severe pneumonia [3]. However, this revision did not deal with the problem of underdiagnosis of obstructive airway disease. Only a few studies have analysed the impact of its implementation, especially in Southeast Asia [14-17].

\section{A prospective study of children hospitalised with pneumonia in Vietnam}

NGUYEN et al. [1] conducted a prospective study of all 4206 children aged 2-59 months who were admitted with a diagnosis of pneumonia to a large secondary care hospital in Vietnam between July 2017 and June 2018. Using the 2014 WHO case management classification, they found that diagnoses of 1758 children (42\%) did not meet the WHO criteria for pneumonia, while 2196 (52\%) met the criteria for pneumonia, and $252(6 \%)$ for severe pneumonia. Only this last, severe pneumonia group should have been admitted for intravenous antibiotics according to the WHO strategy, and the authors conclude that their study suggests unnecessary use of hospital resources and intravenous antibiotics.

This large, well-conducted study offers a real picture of community-acquired pneumonia management in Vietnam. However, its limitations invite discussion. First, the reader assumes that all the 4206 children included in the study received intravenous antibiotics, but in fact NGUYEN et al. [1] do not describe treatment. Secondly, from the 5874 children originally admitted for pneumonia, the study excluded 1515 children who were transferred to a private ward. Thirdly, although we agree that many children with 
pneumonia in this study probably could have been treated as outpatients, as suggested by WHO, some may still have merited hospitalisation and intravenous antibiotics or other specific treatment.

The children in this study were treated in a secondary hospital, nearly all had chest radiograph and full blood count performed, and half had received antibiotics before admission. This is an entirely different scenario from that for which the WHO case management strategies were developed: community healthcare workers with no access to complementary tests. Among the children classified by WHO criteria as not having pneumonia, $37 \%$ had a consolidation on the chest radiograph and $22 \%$ had C-reactive protein $\geqslant 50 \mathrm{mg} \cdot \mathrm{L}^{-1}$. Should these children be classified as "no pneumonia" because they did not present tachypnoea at admission? Given the wide variability of respiratory rate in infants and different states of wakefulness at admission [18, 19], applying the WHO case management criteria in a setting where radiography and full blood counts are readily available seems questionable. What would have happened if the WHO case management criteria had been strictly applied?

Although the highest proportion of adverse outcomes (death, intensive care unit admission, tertiary care transfer or hospital stay $>10$ days) occurred in the group with severe pneumonia $(153 / 252,61 \%)$, in absolute numbers more severe outcomes occurred in the other two groups: without pneumonia $(236 / 1758$, $13 \%)$ and simple pneumonia $(399 / 2196,18 \%)$. These children may not have had severe pneumonia according to the WHO criteria, but some had severe disease. This important study therefore reveals both an overdiagnosis of pneumonia and an underdiagnosis of other potentially fatal diseases like sepsis, cyanotic heart disease or pericarditis (the causes of death of the five children who did not have severe pneumonia according to WHO criteria), and asthma. In such a setting, improving healthcare workers' use of available clinical and complementary tests to identify alternative diagnoses in severely ill children may reduce childhood mortality more effectively than blindly applying WHO algorithms developed for community healthcare workers. This would also decrease the underdiagnosis of other respiratory diseases like asthma and viral wheeze.

\section{Asthma is underdiagnosed and undertreated in low and middle income countries}

According to the 2014 Global Burden of Disease study, 14\% of children worldwide suffer from asthma [20]. Despite wide regional variability [20], asthma is no longer considered a disease of high income countries. In Asia, asthma prevalence is variable with higher prevalence in heavily industrialised countries like Japan and Singapore, and lower prevalence in less industrialised countries like Indonesia and Malaysia [21], with an increasing trend [22]. A study conducted in Hanoi, Vietnam in 1999 estimated a prevalence of current wheeze of $15 \%$ and doctor-diagnosed asthma of $14 \%$ in children 5-11 years old [23]. In 2016, children 6 years old from a birth cohort in a smaller, less polluted city (Nha Trang) had a $5 \%$ prevalence of current wheeze [24].

The implementation of the WHO IMCI guidelines may result in an underdiagnosis of asthma and viral wheeze [11]. In India, $46 \%$ of 200 children aged 6 months to 5 years who presented to the emergency department with cough or difficulty breathing and were initially classified as having pneumonia according to WHO criteria, were finally diagnosed by clinicians as having asthma [25]. Among the children hospitalised for pneumonia in the NGUYEN et al. [1] study, 13\% (550/4206) had audible wheeze and 26\% had wheeze on auscultation. The proportions of wheeze on auscultation were $16 \%, 32 \%$ and $38 \%$, respectively, among those classified as no pneumonia, simple pneumonia, and severe pneumonia. These children most likely had an obstructive airway disease. Treatment is not reported in the study, but these children likely did not receive bronchodilators and corticosteroids, but unnecessary antibiotics instead.

Inadequate treatment results in a high asthma burden in Southeast Asia despite its relatively low prevalence. In 2000, the Asthma Insights and Reality in Asia-Pacific (AIRIAP) study conducted a survey among adults and children with physician-diagnosed asthma from urban centres in eight Asian countries, including Vietnam [26]. Although 50\% of participants reported daytime asthma symptoms and $44 \%$ sleep disturbance, most were not aware of their problems; even among those with severe persistent asthma, 34\% thought their asthma was well-controlled. Only $18 \%$ of those with severe persistent asthma used regular controller medication. Inadequate asthma control increases use of emergency care for acute exacerbations (44\% in the AIRIAP study). This may result in higher asthma mortality compared to countries with higher prevalence but better management. We lack studies on asthma mortality in Southeast Asia, but in Latin American countries asthma control is insufficient and mortality high [27]. CHUA et al. [28] estimated paediatric asthma mortality and hospitalisation trends in 12 Asian Pacific countries. They found that the controller-to-reliever medication ratio was associated with an overall decline in mortality and hospitalisations.

\section{Lessons learned and recommendations}

The WHO case management strategies for pneumonia have certainly helped reduce childhood mortality and intravenous antibiotic use in low resource settings $[9,29]$. However, in secondary or tertiary hospitals 


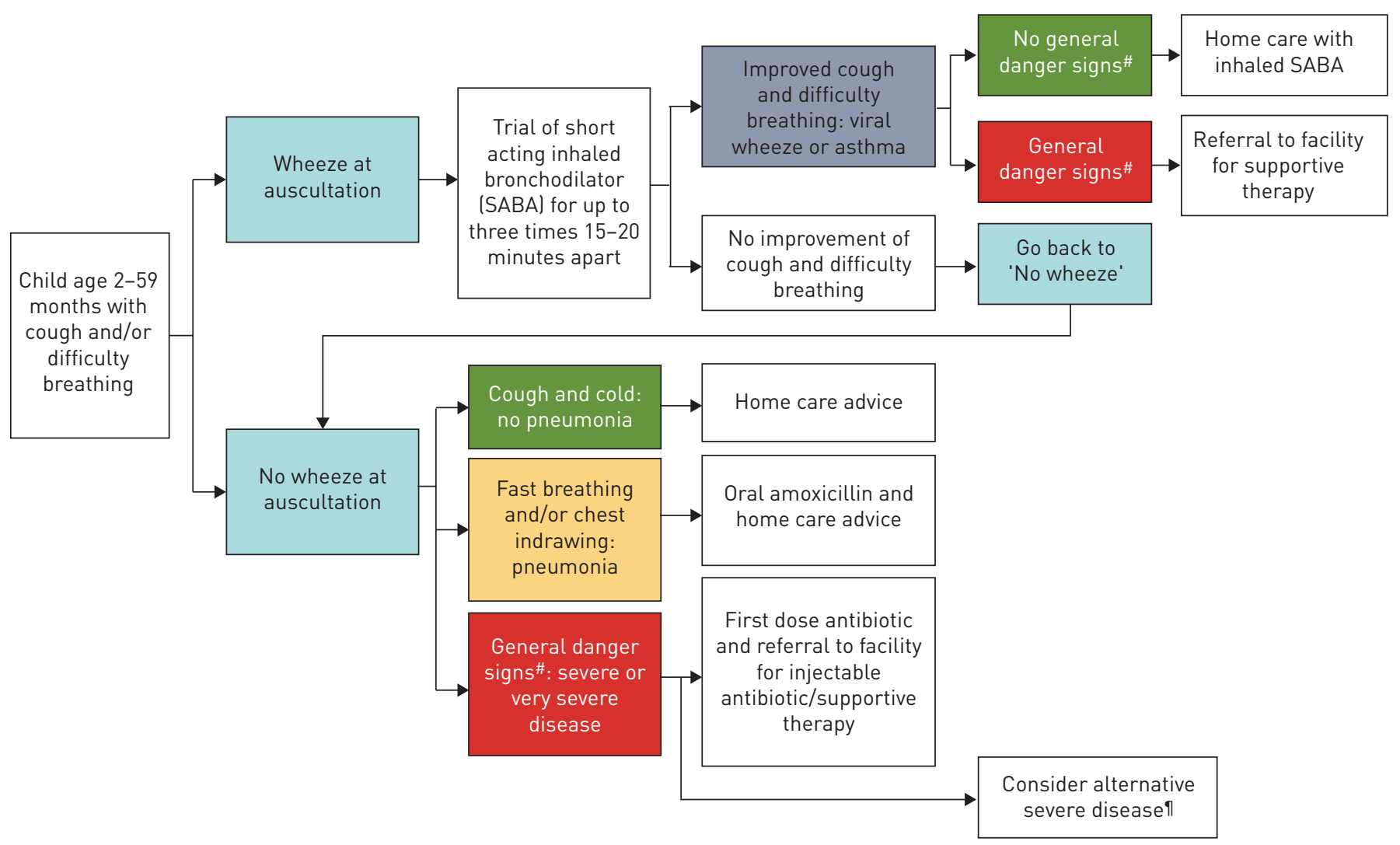

FIGURE 2 Proposed modified algorithm to evaluate children 2-59 months old with cough and/or difficulty breathing. \#: peripheral oxygen saturation $<90 \%$ in room air, severe respiratory distress (grunting or nasal flaring), inability to drink or breastfeed, vomiting everything, lethargy or convulsions; ": sepsis, cyanotic heart disease, pericarditis, other upper or lower respiratory tract diseases.

where trained professionals, radiography and laboratory tests are available, healthcare workers should be trained to identify severely ill children and apply differential diagnosis strategies, rather than strictly applying an algorithm developed for community settings.

We propose that the different respiratory diseases are not dealt with in separate management plans, but are combined into one where alternative diagnoses are considered. In primary care settings, we propose a new algorithm to assess a child with cough and/or difficulty breathing that includes the evaluation of wheeze and response to bronchodilators, and the consideration of alternative diagnoses for severe disease. The algorithm is represented in figure 2.

Finally, we advocate that future epidemiological studies combine assessment of overdiagnosis of pneumonia and underdiagnosis of asthma. They are both current public health problems in LMICs associated with considerable morbidity and mortality. They are related to each other, and studying both in isolation can conceal important consequences of each for the other. Pneumonia, obstructive airway diseases, and other respiratory and nonrespiratory diseases need to be clinically managed and studied together to further reduce childhood mortality, inadequate treatment and waste of healthcare resources.

Acknowledgements: We thank Christopher Ritter (Institute of Social and Preventive Medicine, University of Bern, Switzerland) for his editorial assistance.

Support statement: This work has been supported by a grant from the Swiss National Science Foundation (SNF320030_182628). Funding information for this article has been deposited with the Crossref Funder Registry.

Conflict of interest: None declared.

\section{References}

1 Nguyen PTK, Tran HT, Fitzgerald DA, et al. Characterisation of children hospitalised with pneumonia in central Vietnam: a prospective study. Eur Respir J 2019; 54: 1802256.

2 GBD Compare Data Visualization. http://vizhub.healthdata.org/gbd-compare Date last accessed: April 07, 2019. 
3 World Health Organization. IMCI Integrated Management of Childhood Illness. Department of Child and Adolescent Health and Development. Geneva, World Health Organization, 2005.

4 Zar H, Madhi S, Aston S, et al. Pneumonia in low and middle income countries: progress and challenges. Thorax 2013; 68: 1052-1056.

5 Bradley JS, Byington CL, Shah SS, et al. The management of community-acquired pneumonia in infants and children older than 3 months of age: clinical practice guidelines by the Pediatric Infectious Diseases Society and the Infectious Diseases Society of America. Clin Infect Dis 2011; 53: e25-e76.

6 Harris M, Clark J, Coote N, et al. British Thoracic Society guidelines for the management of community acquired pneumonia in children: update 2011. Thorax 2011; 66: iil-ii23.

7 Zimmerman DR, Kovalski N, Fields S, et al. Diagnosis of childhood pneumonia: clinical assessment without radiological confirmation may lead to overtreatment. Pediatr Emerg Care 2012; 28: 646-649.

8 World Health Organization (WHO). Recommendations for Management of Common Childhood Conditions: Evidence for Technical Update of Pocket Book Recommendations: Newborn Conditions, Dysentery, Pneumonia, Oxygen Use and Delivery, Common Causes of Fever, Severe Acute Malnutrition and Supportive Care. Geneva, World Health Organization, 2012.

9 Sazawal S, Black RE, Group PCMT. Effect of pneumonia case management on mortality in neonates, infants, and preschool children: a meta-analysis of community-based trials. Lancet Infect Dis 2003; 3: 547-556.

10 Theodoratou E, Al-Jilaihawi S, Woodward F, et al. The effect of case management on childhood pneumonia mortality in developing countries. Int J Epidemiol 2010; 39: i155-i171.

11 Østergaard MS, Nantanda R, Tumwine JK, et al. Childhood asthma in low income countries: an invisible killer? Prim Care Respir J 2012; 21: 214.

12 World Health Organization. Revised WHO Classification and Treatment of Pneumonia in Children at Health Facilities: Evidence Summaries. Geneva, World Health Organization, 2014.

13 Nair H, Simoes EA, Rudan I, et al. Global and regional burden of hospital admissions for severe acute lower respiratory infections in young children in 2010: a systematic analysis. Lancet 2013; 381: 1380-1390.

14 Ginsburg AS, Mvalo T, Nkwopara E, et al. Placebo vs amoxicillin for nonsevere fast-breathing pneumonia in Malawian children aged 2 to 59 months: a double-blind, randomized clinical noninferiority trial. JAMA pediatrics 2019; 173: 21-28.

15 Onono M, Abdi M, Mutai K, et al. Community case management of lower chest indrawing pneumonia with oral amoxicillin in children in Kenya. Acta Paediatr 2018; 107: 44-52.

16 Tuti T, Agweyu A, Mwaniki P, et al. An exploration of mortality risk factors in non-severe pneumonia in children using clinical data from Kenya. BMC Med 2017; 15: 201.

17 Ueno F, Tamaki R, Saito M, et al. Age-specific incidence rates and risk factors for respiratory syncytial virus-associated lower respiratory tract illness in cohort children under 5 years old in the Philippines. Influenza Other Respir Viruses 2019.

18 Hoppenbrouwers T, Harper R, Hodgman J, et al. Polygraphic studies of normal infants during the first six months of life. II. Respiratory rate and variability as a function of state. Pediatr Res 1978; 12: 120.

19 Muro F, Mtove G, Mosha N, et al. Effect of context on respiratory rate measurement in identifying non-severe pneumonia in African children. Trop Med Int Health 2015; 20: 757-765.

20 Lai CK, Beasley R, Crane J, et al. Global variation in the prevalence and severity of asthma symptoms: phase three of the International Study of Asthma and Allergies in Childhood (ISAAC). Thorax 2009; 64: 476-483.

21 Wong GW, Leung TF, Ko FW. Changing prevalence of allergic diseases in the Asia-pacific region. Allergy Asthma Immunol Res 2013; 5: 251-257.

22 Pearce N, Ait-Khaled N, Beasley R, et al. Worldwide trends in the prevalence of asthma symptoms: phase III of the International Study of Asthma and Allergies in Childhood (ISAAC). Thorax 2007; 62: 758-766.

23 Nga NN, Chai SK, Bihn TT, et al. ISAAC-based asthma and atopic symptoms among Ha Noi school children. Pediatr Allergy Immunol 2003; 14: 272-279.

24 Toizumi M, Hashizume M, Nguyen HAT, et al. Asthma, rhinoconjunctivitis, eczema, and the association with perinatal anthropometric factors in Vietnamese children. Sci Rep 2019; 9: 2655.

25 Sachdev HP, Mahajan SC, Garg A. Improving antibiotic and bronchodilator prescription in children presenting with difficult breathing: experience from an urban hospital in India. Indian Pediatr 2001; 38: 827-838.

26 Lai CK, De Guia TS, Kim YY, et al. Asthma control in the Asia-Pacific region: the Asthma Insights and Reality in Asia-Pacific Study. J Allergy Clin Immunol 2003; 111: 263-268.

27 Neffen H, Baena-Cagnani CE, Malka S, et al. Asthma mortality in Latin America. J Investig Allergol Clin Immunol 1997; 7: 249-253.

28 Chua KL, Soh SE, Ma S, et al. Pediatric asthma mortality and hospitalization trends across Asia pacific: relationship with asthma drug utilization patterns. World Allergy Organ J 2009; 2: 77-82.

29 Qazi S, Rehman G, Khan M. Standard management of acute respiratory infections in a children's hospital in Pakistan: impact on antibiotic use and case fatality. Bull World Health Organ 1996; 74: 501-507. 